\title{
Extramedullary Multiple Myeloma Mimicking Liver Metastases during Carfilzomib Treatment
}

\author{
Mensah C* and Osunkwo I \\ Department of Hematology, North Shore University \\ Hospital, USA \\ *Corresponding author: Cheryl Mensah, Department \\ of Hematology, North Shore University Hospital, 300 \\ Community Dr, Manhasset, NY 11030, USA
}

Received: January 17, 2017; Accepted: March 09, 2017; Published: March 20, 2017

\begin{abstract}
Multiple myeloma accounts for $1.9 \%$ of all cancer deaths. The median overall survival is seven to ten years in standard risk patients. However, high risk myeloma patients have median overall survival of three to five years. Features that stratify patients to high risk include: advanced stage disease, chromosomal abnormalities, plasma cell leukemia, and extramedullary disease. The prevalence of hepatic extra osseous disease has not been established and published data is limited to autopsy and case reports. There is no standard treatment for extra osseous myeloma. Proteasome Inhibitors (PI) and Immunomodulatory Derivatives (IMID) such as thalidomide, lenalidomide, and pomalidomide have led to improved overall survival in multiple myeloma. However, even with these new therapeutic agents, extramedullary disease is associated with poor prognosis and reduced overall survival. In addition, there has been increased incidence of treatment emergent extramedullary disease after treatment with novel agents. The following case report describes an uncommon manifestation of multiple myeloma discovered during treatment with novel agents.
\end{abstract}

\section{Introduction}

The National Cancer Institute (NCI) estimates that 12,650 people died from multiple myeloma in 2016 [1]. Currently, multiple myeloma accounts for $2.1 \%$ of all cancer deaths in the United States. The median overall survival is seven to ten years in standard risk patients. However, high risk myeloma patients have a median overall survival of three to five years [2]. Features that stratify patients to high risk include: advanced stage disease, chromosomal abnormalities, plasma cell leukemia, and extramedullary disease $[3,4]$. Most multiple myeloma patients have malignant plasma cells confined to the bone marrow and the skeleton. The mechanism of development of extraosseous spread is not well understood. The prevalence of hepatic extraosseous disease has not been established and published data is limited. This case report describes an uncommon manifestation of multiple myeloma. Following our case, we included a literature review of extramedullary myeloma in patients treated with novel agents.

\section{Case Description}

In August 2014, a 53-year-old man presented to the Emergency Department (ED) with a chief complaint of back pain. The back pain was in the lumbosacral region, 10 out of 10 in severities, constant, unrelieved by naproxen and methocarbamol, lasting for 3 weeks' duration. Initial workup with a basic metabolic panel and complete blood count revealed a normocytic anemia, hyperproteinemia, and hypoalbuminemia. Hemoglobin $(\mathrm{Hgb})$ was $11.0 \mathrm{~g} / \mathrm{dl}$, total protein $11.1 \mathrm{gm} / \mathrm{dl}$, and albumin was $2.0 \mathrm{gm} / \mathrm{dl}$. Calcium was within normal limits at $9.7 \mathrm{mg} / \mathrm{dl}$. Serum Blood Urea Nitrogen (BUN) was $33 \mathrm{mg} / \mathrm{dl}$ and creatinine was $2.77 \mathrm{mg} / \mathrm{dl}$ with no known baseline of comparison. The patient was discharged from the ED and advised to follow up with a primary care physician. The following day, patient returned to the ED with chief complaints of persistent back pain, new cough, and body aches. Laboratory tests demonstrated normocytic anemia, worsening hyperproteinemia with total protein $12.8 \mathrm{~g} / \mathrm{dl}$, and hypoalbuminemia of $2.6 \mathrm{gm} / \mathrm{dl}$. The patient was found have acute renal failure with a BUN of $38 \mathrm{mg} / \mathrm{dl}$ and creatinine $(\mathrm{Cr}) 3.59 \mathrm{mg} / \mathrm{dl}$. Chest roentgenogram found a right lower lobe infiltrate. The patient was admitted to the hospitalist medicine service for treatment of community acquired pneumonia.

During the admission, a serum protein electrophoresis was performed and the patient was found to have a serum monoclonal protein of $2.8 \mathrm{~g} / \mathrm{dl}$. A skeletal survey found numerous small lytic lesions from the calvarium throughout most of the spine. Bone marrow biopsy found $60 \%$ plasma cells in the bone marrow. A urine immunofixation demonstrated an IgG Kappa band, Bence Jones Protein kappa type. The Beta 2 microglobulin was $7.4 \mathrm{mg} / \mathrm{L}$ and the albumin was $2.3 \mathrm{~g} / \mathrm{dl}$. The patient was diagnosed with IgG kappa multiple myeloma stage III per International Staging System (ISS) Criteria. The patient was discharged with dexamethasone and advised to follow up with a hematologist.

At the hematologist office, the patient had a normal karyotype on cytogenetic analysis. The Fluorescence in situ Hybridization (FISH) multiple myeloma panels were negative. Based on the patient's age of less than 60 years and normal cytogenetics, the patient was treated as standard risk with the possibility of a future bone marrow autologous transplant. Bortezomib, Cyclophosphamide, Dexamethasone (VCD) treatment was initiated with bortezomib $1.5 \mathrm{mg} / \mathrm{m}^{2}$ subcutaneous weekly, cyclophosphamide $300 \mathrm{mg} / \mathrm{m}^{2}$ orally, and dexamethasone $40 \mathrm{mg}$ orally on days $1,8,15$, and 22 . The patient completed four cycles of VCD treatment.

During treatment in January 2015, a repeat bone marrow biopsy and aspiration was performed. Patient had normal trilineage hematopoiesis and scattered plasma cells. Plasma cells were less than five percent. After treatment in February 2015, the serum monoclonal 
Table 1: The literature review of 12 articles.

\begin{tabular}{|c|c|c|c|c|c|}
\hline Reference & Type & $\begin{array}{l}\text { Number of } \\
\text { Patients }\end{array}$ & Treatment & $\begin{array}{c}\text { Liver lesions } \\
\text { discovered during or after } \\
\text { Novel Agent }\end{array}$ & $\begin{array}{c}\text { Biopsy Proven Liver } \\
\text { Lesion }\end{array}$ \\
\hline Rahhal et al [7] & Case Report & 1 & $\begin{array}{c}\text { TD } \\
\text { then Bortezomib }\end{array}$ & yes & yes \\
\hline Islam et al [8] & Case Report & 1 & PAD & no & no \\
\hline Lopes et al [9] & Case Report & 1 & $\begin{array}{l}\text { VAD then Melphalan then AutoSCT then } \\
\text { Thalidomide then } \\
\text { Vel/Dex }\end{array}$ & yes & yes \\
\hline Coffey et al [10] & Case Report & 1 & RVD & no & yes \\
\hline Tan et al [11] & Case Report & 1 & VTD & no & yes \\
\hline Wilson et al [12] & Case Report & 1 & CyBorD then Vel/Dex then AutoSCT & no & yes \\
\hline De Vos et al [13] & Case Report & 1 & CyBorD then MTD then Lenalidomide & no & yes \\
\hline Sekiguchi et al [14] & Case Report & 1 & $\begin{array}{l}\text { CHOP then VCAP then TD then Vel/dex then } \\
\text { Methylprednisolone }\end{array}$ & no & no \\
\hline Ise et al [16] & Case Report & 1 & Vel/Dex then VTD then Melphalan then RD & yes & yes \\
\hline Jha et al [17] & Case Report & 1 & CyBorD & no & yes \\
\hline Brilland et al [6] & Case Report & 1 & Vel/Dex & yes & yes \\
\hline
\end{tabular}

TD: Thalidomide/Dexamethasone; PAD: Bortezomib/Doxorubicin/Dexamethasone; VAD: Vincristine/Doxorubicin/Dexamethasone; AutoSCT: Autologous Stem Cell Transplant; Vel/dex: Bortezomib/Dexamethasone; RVD: Lenalidomide/Bortezomib/Dexamethasone; VTD: Bortezomib/Thalidomide/Dexamethasone; CyBorD: Cyclophosphamide/Bortezomib/Dexamethasone; MTD: Melphalan/Thalidomide/Dexamethasone; CHOP: Cyclophosphamide/Adriamycin/Vincristine/Prednisone; VCAP: Vincristine/Cyclophosphamide/Adriamycin/Prednisone; RD: Lenalidomide/Dexamethasone; MP: Melphalan/Prednisone; MD: Melphalan/Dexamethasone; VMCP: Vincristine/Melphalan/Cyclophosphamide/Prednisone

protein was $0.2 \mathrm{~g} / \mathrm{dl}$ on serum protein electrophoresis. However, an IgG Kappa band was identified on serum immunofixation. Patient achieved a very good partial response and was referred for autologous stem cell transplant. Other laboratory findings demonstrated resolution of normocytic anemia and renal failure with $\mathrm{Hgb}$ of $15.3 \mathrm{~g} /$ $\mathrm{dl}$ and $\mathrm{Cr}$ of $0.89 \mathrm{mg} / \mathrm{dl}$. Calcium remained in normal limits at $9.6 \mathrm{mg} /$ dl. Free light chain ratio was within normal limits at 1.35 . Treatment with zoledronic acid was initiated for his lytic lesions.

In June 2015, the patient finally agreed to attend pre-testing appointments for autologous stem cell transplant. However, this was interrupted when he was hospitalized for community acquired pneumonia. This hospitalization was complicated by acute renal failure, hypercalcemia, and anemia. On this admission, the BUN was $31 \mathrm{mg} / \mathrm{dl}$, Cr was $4.09 \mathrm{mg} / \mathrm{dl}$, calcium was $11.0 \mathrm{mg} / \mathrm{dl}$, and ionized calcium was $1.38 \mathrm{mmol} / \mathrm{L}$. The patient received antibiotics, intravenous fluids, and dexamethasone. $\mathrm{Cr}$ improved to $1.09 \mathrm{mg} /$ $\mathrm{dl}$ and calcium normalized to $8.6 \mathrm{mg} / \mathrm{dl}$. The normocytic anemia persisted with an $\mathrm{Hgb}$ of $9.9 \mathrm{~g} / \mathrm{dl}$. Patient discharged and instructed to follow up with the hematologist.

At the hematologist office in July 2015, a repeat serum monoclonal protein had risen to $2.9 \mathrm{~g} / \mathrm{dl}$ on a serum protein electrophoresis. Patient had progression of disease and the decision was made to start (RD) lenalidomide and dexamethasone. Unfortunately, less than a week later, patient returned to the hospital with chief complaints of altered mental status, weakness, and subjective fevers. Patient was found to have a worsening normocytic anemia, acute renal failure with metabolic acidosis, hyperkalemia, and hypercalcemia. Hgb was $7.0 \mathrm{~g} / \mathrm{dl}, \mathrm{BUN}$ was $62 \mathrm{md} / \mathrm{dl}, \mathrm{Cr}$ was $10.21 \mathrm{mg} / \mathrm{dl}$, potassium was 6.9 $\mathrm{mmol} / \mathrm{L}$, and calcium was $11.2 \mathrm{mg} / \mathrm{dl}$. Patient was admitted to the medical intensive care unit for urgent hemodialysis.

Serum kappa free light chains were $1,180 \mathrm{mg} / \mathrm{dl}$ and serum lambda free light chains were $0.64 \mathrm{mg} / \mathrm{dl}$. Kappa Lambda free light chain ratio was $1,843.75$ significantly increased from 1.35 post chemotherapy six months ago. Hematology was consulted in the hospital. High dose dexamethasone was started at 40mg. Decision was made to treat a likely myeloma cast nephropathy with carfilzomib and plasmapheresis.

Patient completed five sessions of plasmapheresis. Also, he received carfilzomib $15 \mathrm{mg} / \mathrm{m}^{2}$ on day 1, 2, 8, and 9. Serum Kappa free light chains decreased to $270.0 \mathrm{mg} / \mathrm{dl}$ and serum lambda free light chains were $0.77 \mathrm{mg} / \mathrm{dl}$ post plasmapheresis. Kappa Lambda free light chain ratio also decreased to 350.65 . The patient did not have day 15 and 16 carfilzomib, thus he did not complete cycle 1 . Unfortunately, he developed transaminitis with a hyperbilirubinemia. Aspartate aminotransferase (AST) was $114 \mathrm{U} / \mathrm{L}$, Alanine aminotransferase (ALT) was $82 \mathrm{U} / \mathrm{L}$, Serum alkaline phosphatase was $605 \mathrm{U} / \mathrm{L}$, and total serum bilirubin was $7.0 \mathrm{mg} / \mathrm{dl}$. An MRI abdomen without contrast found innumerable hepatic nodules suspicious for metastases.

A needle core biopsy into the right liver lesion found liver tissue infiltrated with sheets of atypical plasma cells. The plasma cells were positive for CD138, kappa light chain, and CD56 on immunohistochemistry consistent with a plasma cell neoplasm in the liver. The patient was diagnosed with extramedullary multiple myeloma in the liver. The decision was made to treat the patient with Dexamethasone, Cyclophosphamide, Etoposide, and Cisplatin (DCEP) for refractory and treatment emergent extramedullary multiple myeloma.

\section{Literature Review}

We performed a literature review on hepatic extraosseous multiple myeloma in patients treated with novel agents. First we searched the electronic MEDLINE database using the MeSH search terms "multiple myeloma" AND "liver." Using these terms we had 
246 hits. Among those hits, we used articles that met the following inclusion criteria: full text, English published, human only studies and reports. We allowed myeloma related diseases such as light chain deposition disease and plasma cell leukemia. We also included articles with myeloma related liver manifestations such as plasmacytomas and/or liver light chain deposition.

We excluded articles with the following criteria: liver disease as a result of treatment, liver disease as a result of alternate malignancy, or liver disease due to nonmalignant conditions such as amyloidosis, hepatitis, etc. We excluded amyloidosis cases because hepatic involvement is a common complication of that disease [5]. Using these criteria, we found 31 articles describing extraosseous myeloma involvement in the liver on MEDLINE. To expand our list, we searched MEDLINE using multiple MeSH search terms: "multiple myeloma" AND "extramedullary hematopoiesis", "extraosseous" AND "multiple myeloma", "plasmacytoma" AND "multiple myeloma" AND "liver". Unfortunately, those MeSH searches did provide articles that met our inclusion criteria.

To expand our search, we used MeSH terms "plasmacytoma" and "multiple myeloma" which gave 1701 hits on MEDLINE. Among those hits, we included full text articles published within the last 10 years narrowing our hits to 248 . We preferred articles within the last 10 years because thalidomide, the earliest novel agent, was FDA approved for myeloma in 2006 [6]. We excluded articles that primarily referred to other anatomic locations in the title such as pulmonary, testis, etc. Among the articles remaining, we searched for "liver" or hepatic" within the full text. Using these additional methods, we expanded our total article number to 42 .

Within those 42 articles, we added an additional inclusion criterion: treatment with novel agents. We looked for articles using novel agents such as immunomodulatory derivatives (IMID), proteasome inhibitors, histone deacetylase inhibitors, monoclonal antibodies, etc. We found only 12 articles that describe treatment or diagnosis of hepatic extramedullary myeloma with these novel agents. We also searched the MEDLINE database using the MeSH search terms: "multiple myeloma" AND "liver" AND "carfilzomib". There was only one hit and that article did not describe a hepatic manifestation of myeloma. Based on this literature review using MEDLINE, our case is the only case of hepatic extraosseous multiple myeloma during carfilzomib treatment.

Below is a table of the 12 articles found in our literature review? These articles met our inclusion criteria as: full text, English published human only, hepatic manifestation of multiple myeloma, treated with a novel agent (Table 1).

\section{Conclusion}

Extraosseous myeloma is rare at diagnosis and more common in refractory disease or high risk myeloma patients [18]. In a case series of 1027 patients, the incidence of extramedullary disease at diagnosis was only $0.4 \%$ [19]. However during the course of disease or at relapsed, an estimated 6-20\% of patients develop extramedullary manifestations of myeloma [20].

Our patient, despite normal cytogenetics, had high risk myeloma due to his advanced ISS stage III disease. In addition, his extramedullary disease developed during relapse after his initial treatment with a novel agent. Per the International Myeloma Working Group, high risk disease is treated aggressively with a threedrug regimen followed by autologous transplant [4]. These three drug regimens typically include immunomodulatory agents, proteasome inhibitors, and steroids. The use of an alkylating agent is minimized to prevent drug resistance and clonal evolution [4]. Our patient was initially treated with a proteasome inhibitor, alkylating agent and a steroid and achieved a very good response. Despite this response, the patient developed relapsed disease and myeloma kidney. The relapsed disease was treated with a newer generation proteasome inhibitor, carfilzomib. During this treatment, the patient developed treatment emergent extraosseous disease in the liver.

The factors that lead to extraosseous myeloma are unclear. It's postulated that cell adhesion molecule expression is responsible for the spread of multiple myeloma cells outside of the bone marrow [21]. Particularly cell adhesion molecules CD44 and CD56 which mediate myeloma cell adhesion to bone marrow stroma. Galani et al., theorized that cell adhesion molecule expression changes during the course of disease [21]. In 2012, Usmani et al. published that the incidence of extramedullary myeloma was increased in patients treated with novel agents at their Multiple Myeloma Institute in Arkansas [22]. Their findings added evidence to the belief that novel agents increase the risk of extraosseous development. However in 2015, Varga et al. examined the use of novel agents such as lenalidomide/ bortezomib and the development of extraosseous myeloma at the Dana Farber Cancer Institute. Varga et al. found no increased risk of extramedullary disease using those novel agents, further complicating our understanding [20].

Currently, there is no consensus on treatment of extramedullary myeloma. Proteasome Inhibitors (PI) and Immunomodulatory Derivatives (IMID) such as thalidomide, lenalidomide, and pomalidomide have led to improved overall survival in multiple myeloma. However, even with these new therapeutic agents, extramedullary disease is associated with poor prognosis and reduced overall survival [5]. Perhaps with more cases and further use of the 2015 newly approved agents such as Ixazomib, Daratumumab, and Elotuzumab we may see improved outcomes for this deadly disease.

\section{References}

1. SEER Cancer Statistics Factsheets: Myeloma. National Cancer Institute. Bethesda M.

2. Barkay I, Maziarz RT, Chen Al, Dibb W, Chen Y, Scott EC. Extramedullary Multiple Myeloma Associated with Reduced Overall Survival: A Retrospective Single-Center Study. Blood. 2013; 122: 5317.

3. Lonial S, Boise LH, Kaufman J. How I treat high-risk myeloma. Blood. 2015; 126: 1536-1543.

4. Usmani SZ, Heuck C, Mitchell A, Szymonifka J, Nair B, Hoering A, et al. Extramedullary disease portends poor prognosis in multiple myeloma and is over-represented in high-risk disease even in the era of novel agents. Haematologica. 2012; 97: 1761-1767.

5. Galani Z, Viniou N, Kyrtsonis MC, Sachanas S, Antoniou T, Dimopoulou MN, et al. Hepatic and renal plasma cell lesions in a patient with multiple myeloma in hematological remission. Anticancer Res. 2007; 27: 571-574.

6. FDA Approves Thalomid (thalidomide) to Treat Multiple Myeloma. US Food and Drug. 2006.

7. Islam MS, Anoop P. Images in hematology. Transient erythrocyte changes 
caused by infiltration of liver by plasma cell leukemia. Am J Hematol. 2011; 86: 67-68.

8. Lopes da Silva R, Monteiro A, Veiga J. Non-secretory multiple myeloma relapsing as extramedullary liver plasmacytomas. J Gastrointestin Liver Dis. 2011; 20: 81-83.

9. Coffey D, Fain B, Thompson C, Chan ED, Nawaz S. Liver failure as the only clinical manifestation of multiple myeloma. Ann Hematol. 2012; 91: 625-627.

10. Tan $\mathrm{CH}$, Wang $\mathrm{M}$, Fu WJ, Vikram R. Nodular extramedullary multiple myeloma: hepatic involvement presenting as hypervascular lesions on CT. Ann Acad Med Singapore. 2011; 40: 329-331.

11. Wilson N, Surati M, Walker BF, Kaufman JL, Harvey RD. Bortezomib administration with severe hyperbilirubinemia caused by hepatic plasma cell infiltration: a case report. Clin Lymphoma Myeloma Leuk. 2013; 13: 159-161.

12. de Vos M, Druez P, Nicaise M, Ngendahayo P, Sinapi I, Mineur P. Multiple myeloma presenting as hepatic nodular lesion. Acta Clin Belg. 2012; 67: 378380.

13. Sekiguchi $Y$, Asahina $T$, Shimada $A$, Imai $H$, Wakabayashi $M$, Sugimoto $K$ et al. A case of extramedullary plasmablastic plasmacytoma successfully treated using a combination of thalidomide and dexamethasone and a review of the medical literature. J Clin Exp Hematop. 2013; 53: 21-28.

14. Pal S, Chattopadhyay B1, Chatterjee A, Bhattacharya B. Lambda light chain myeloma presenting as nodular hepatic lesion: a clinical rarity. J Cancer Res Ther. 2014; 10: 191-193.

15. Ise M, Tsujimura H, Sakai C, Kumagai K. Hepatic extramedullary disease in multiple myeloma with 17p deletion. Clin Lymphoma Myeloma Leuk. 2014 14: e165-168.
16. Jha LK, Dingli D. An unusual cause of ascites. Blood. 2015; 126: 1629.

17. Brilland B, Sayegh J, Croue A, Bridoux F, Subra JF, Augusto JF. Recovery from LCDD-associated Severe Liver Cholestasis: a Case Report and Literature Review. J Gastrointestin Liver Dis. 2016; 25: 99-103.

18. Moon YS. Hepatic amyloidosis. Korean J Hepatol. 2011; 17: 80-83.

19. Short KD, Rajkumar SV, Larson D, Buadi F, Hayman S, Dispenzieri A, et al. Incidence of extramedullary disease in patients with multiple myeloma in the era of novel therapy, and the activity of pomalidomide on extramedullary myeloma. Leukemia. 2011; 25: 906-908.

20. Varettoni M, Corso A, Pica G, Mangiacavalli S, Pascutto C, Lazzarino M. Incidence, presenting features and outcome of extramedullary disease in multiple myeloma: a longitudinal study on 1003 consecutive patients. Ann Oncol. 2010; 21: 325-330.

21. Rahhal F, Schade R, Nayak A, Coleman TA. Hepatic failure caused by plasma cell infiltration in multiple myeloma. World J Gastroenterol. 2009; 15 : 2038-2040.

22. Varga C, Xie W, Laubach J, Ghobrial IM, O'Donnell EK, Weinstock M, et al. Development of extramedullary myeloma in the era of novel agents: no evidence of increased risk with lenalidomide-bortezomib combinations. $\mathrm{Br} \mathrm{J}$ Haematol. 2015; 169: 843-850.
J Blood Disord - Volume 4 Issue 1 - 2017

ISSN 2379-8009 | www.austinpublishinggroup.com

Mensah et al. @ All rights are reserved
Citation: Mensah C and Osunkwo I. Extramedullary Multiple Myeloma Mimicking Liver Metastases during Carfilzomib Treatment. J Blood Disord. 2017; 4(1): 1041. 